\title{
Controlling low-temperature tunneling dynamics with external fields
}

\author{
M. Morillo* and R. I. Cukier \\ Department of Chemistry and Center for Fundamental Materials Research, Michigan State University, \\ East Lansing, Michigan 48824-1322
}

(Received 23 October 1995; revised manuscript received 8 August 1996)

\begin{abstract}
The tunneling of a particle between two states, as influenced by coupling the particle to an external field, is investigated. A spin-boson Hamiltonian is used to describe the tunnel system and its interaction with a medium and is augmented by a term coupling the dipole moment of the particle to the external field. A projection operator method is introduced that provides equations of motion for the particle's density matrix and permits discussion of external field effects on tunneling even at very low temperatures. These equations of motion can exhibit solutions characterized by population relaxation with a rate constant that is an average over the period of the external field. As an application of this theory, the modification by an external field of the tunneling rate of a defect in a metal, where the system-medium interaction is characterized by an Ohmic spectrum, is evaluated. At sufficiently low temperatures, for experimentally relevant parameter values, suitably chosen (constant and/or sinusoidal), external fields can greatly suppress or enhance the tunneling rate relative to the field-independent value. [S0163-1829(96)06943-3]
\end{abstract}

\section{INTRODUCTION}

Reactions that are based on tunneling find application in diverse areas of biology, chemistry, and physics. ${ }^{1-9}$ Examples of tunneling species include protons, hydrogen, and heavier atoms, chemical groups such as a methyl group, electrons, and defects and impurities in solids. The major emphasis of studies of such reactions has been on characterizing their rates in terms of the given properties of the tunnel system, and the surrounding medium to which the tunnel system is coupled. Recently, there have been works that discuss the possibility of attempting to control tunneling by the application of external fields. ${ }^{10-29}$ If tunneling can be controlled externally, then the possibility of storing and retrieving information can be contemplated. ${ }^{10-12,30-32}$

Our objective is an investigation of the prospect of controlling tunneling by the application of an external field. Tunneling is responsible for the transfer of a "particle" from one spatially localized region to another by the quantummechanical process of wave-function penetration of a potential-energy barrier. ${ }^{33}$ As is well known, ${ }^{33}$ the probability of tunneling increases as the particle's mass decreases, and the height and width of the barrier of the potential decrease and maximize for a symmetric potential profile. At the most basic level, an external field can control tunneling by biasing the potential profile. For example, if the potential profile experienced by the tunnel particle were symmetric, in the absence of an external field, then its application could provide an asymmetry that would greatly reduce the possibility of tunneling. Conversely, an asymmetric potential profile in the absence of an external field could be symmetrized by its application and greatly enhance the tunneling. As a tunneling probability is strongly dependent on the degree of asymmetry in the potential, external fields can have dramatic effects on the tunneling.

In order to control tunneling externally, the tunnel particle must be connected to the external field. Often, tunnel systems exhibit different charge distributions in their different localized states, and therefore an appropriate coupling would be via an electric field. For a charge-neutral tunnel system, the coupling then is between the dipole of the particle and the electric field. Other couplings can be addressed. For example, if the tunnel particle is best described as a hydrogen atom, the coupling would be to an external pressure field. In view of the flexibility of electric fields, with regard to intensity and frequency, and the prevalence of tunnel systems that exhibit significant charge displacement on transfer, electric fields should be viewed as prime candidates for controlling tunneling.

In general, a discussion of tunneling should be carried out from the perspective of the characteristics of the potentialenergy surface that the particle experiences. However, as is typically the case, a reduction to a two-level system is appropriate. Then the problem can be formulated in terms of a spin-boson Hamiltonian ${ }^{3,5,8}$ with the addition of a coupling term to the external field.

In previous work we studied the control of tunneling when the medium could be treated classically in the sense that the $\beta h \omega \ll 1$, where $\beta=1 / k_{B} T$ and $\omega$ is a characteristic medium frequency. ${ }^{13,14}$ The coupling to various forms of an external field could produce dramatic enhancements or reductions of the tunnel rate, when a rate regime was found to be appropriate. We actually focused on the equations of motion of the tunnel system, so that the entire dynamic process could be discussed for different initial conditions. This contrasts with the approach of directly obtaining an approximation to the population evolution by, e.g., a golden rule calculation. This latter approach is directed toward obtaining a rate constant for the rate of population decay. While this is often the regime that emerges in the presence of coupling to a medium, we found examples where the approach to an equilibrium state from some prepared initial state is not monotonous. When the external field is time dependent, an equation of motion method should be used, as golden rule calculations rely on short-time approximations that may be violated in general. 
In this work we consider the situation where $\beta h \omega$ can be large, so that the medium must be treated quantum mechanically. Several groups ${ }^{21,22,26-29}$ considered external field effects in this regime by use of a noninteracting-blip approximation approach. The role of the external field in modifying the transition temperature that separates coherent from incoherent tunneling was examined. As is known from tunneling calculations in this temperature regime, more particulars of the medium enter the theory than at high temperature. In particular, a thermally weighted average of the spectral density characterizing the coupling between the medium and tunnel system enters, which arises in spin-boson Hamiltonian theories, ${ }^{5,8,34}$ in contrast to high temperatures, where only a moment of the spectral density, the reorganization energy, is involved. Nevertheless, the control of tunneling expressions we will develop will be qualitatively the same as the simpler classical medium expressions.

A particular motivation for this investigation is recent experiments on the tunneling of individual defects in submicrometer Bi wires. ${ }^{35-37}$ The low-temperature tunnel dynamics of this defect in bismuth can be modeled as that of a two-level system coupled to a dissipative medium formed from the metal's conduction electrons. ${ }^{38,39}$ At very low temperature, the defect would tunnel coherently between its two available states. As the temperature is increased, a regime is reached where the defect tunnels incoherently, and its dynamics can be described as an exponential decay of the population of an initially prepared state, as described by a rate constant, ${ }^{5,8}$ Kondo $^{38,39}$ noted that, since the electrons are excited in electron-hole pairs around the Fermi level, excitation is possible even for vanishing energies. Consequently, the spectrum characterizing the defect-bath coupling is Ohmic. For symmetric tunneling (the equilibrated states of the defect have equal energy), the rate constant $\sim T^{2 \alpha-1}$. Here $\alpha<\frac{1}{2}$ is a system-bath coupling constant. ${ }^{40}$ This unusual dependence of an increasing rate with decreasing temperature is a consequence of the Ohmic excitation spectrum and the relatively weak coupling of the impurity to the bath. Experimental evidence of this temperature dependence has been found for muon diffusion in $\mathrm{Cu}$ and $\mathrm{Al}^{41}$ and for $\mathrm{H}$ interstitials trapped by $\mathrm{O}$-atom impurities in $\mathrm{Nb}(\mathrm{OH})_{x} \cdot{ }^{42}$ For an asymmetric tunnel system, with asymmetry $\varepsilon$, the behavior is more complex. It depends on whether the rate corresponds to a downhill (exothermic) or uphill (endothermic) process and the temperature regime $\left(k_{B} T<\varepsilon\right.$ or $\left.k_{B} T>\varepsilon\right){ }^{8,43-46}$ Evidence of this complex $T$ dependence has been found in the abovenoted tunneling of individual defects in submicrometer $\mathrm{Bi}$ wires. ${ }^{35-37}$

In order to investigate controlling tunneling when the medium should be treated quantum mechanically, we will use a projection operator method, ${ }^{47,48}$ as the low temperature requires a special treatment. In particular, the tunnel splitting, which characterizes the energy difference between the isolated tunnel states, is no longer suitable as the natural expansion parameter. The Hamiltonian should first be rearranged into a form suitable for carrying out perturbation theory valid at low temperature. The result of our calculations is a set of equations of motion for the system density-matrix elements with coefficients specified in terms of the spectral density, and the effects arising from the coupling to the external field. A constant external field acts simply as another source of asymmetry $\varepsilon$. When the external field is time dependent there is no rate constant, strictly speaking, but we are able to derive useful expressions for an external-field-periodaveraged rate constant. This averaged rate constant expression shows that the rate of tunneling can be controlled by application of periodic and/or constant external fields even at low temperature.

The plan of the remainder of the paper is as follows: In Sec. II we use the projection operator method to obtain the equations of motion for the elements of the tunnel system's density matrix. The external field induces complications in the equations of motion due to noncommutativity of operators taken at different times. It also produces differences in the equations of motion we obtain by the projection operator method and what would be obtained by a generalization of Redfield theory to time-varying external fields. In Sec. III, an approximation of the equations of motion is obtained that leads to a relatively simple evolution equation for the population of an initially localized state. Conditions under which a rate constant description of the relaxation to equilibrium are found, the time-dependent rate (induced by a timevarying external field) is analyzed, and it is averaged over a cycle of the external field to provide the rate constant for an averaged decay of population description. The temperature dependence of this averaged decay constant is analyzed to contrast it with the tunnel rate in the absence of an external field. Our concluding remarks are presented in Sec. IV.

\section{PROJECTION OPERATOR DERIVATION OF THE EQUATIONS OF MOTION}

The Hamiltonian appropriate to a two-level system (TLS) linearly coupled to an infinite number of bosonic excitations with the addition of an external field that couples to the transition dipole of the tunneling object is ${ }^{3,5,49}$

$$
H=V s_{z}+\sum_{j}\left[\frac{p_{j}^{2}}{2}+\frac{1}{2} \omega_{j}^{2}\left(q_{j}-\frac{\gamma_{j}}{\omega_{j}^{2}} s_{x}\right)^{2}\right]+2 b(t) s_{x}
$$

Here $V$ is the splitting of the tunnel doublet in the absence of coupling to the medium; the bath oscillators are characterized by the coordinates and momenta $q j$ 's and $p j$ 's, respectively; and the masses of the oscillators are set to 1 for convenience. The TLS medium couplings are characterized by the $\gamma j$ 's, and the external field's form is left general, for now, as $b(t)$ (the factor of 2 is included for notational convenience). The spin operators, $s_{x}, s_{y}$, and $s_{z}$ are the conventional Pauli matrices. The above Hamiltonian is written on the basis of the tunnel doublet eigenstates $|0+\rangle$ and $|0-\rangle$ of the unperturbed system. The coupling $V$ is responsible for the tunneling between the left $|L\rangle$ and right $|R\rangle$ states, which are defined as the symmetric and antisymmetric linear combinations of the tunnel states: $|L\rangle=(1 / \sqrt{2})[|0+\rangle+|0-\rangle]$ and $|R\rangle=(1 / \sqrt{2})[|0+\rangle-|0-\rangle]$. The problem can be formulated on a localized basis as well; it is purely a matter of convenience as to which basis is used. The origin of the $b(t)$ term is the coupling of an external electric field to the transition dipole moment of the tunneling object. In the delocalized basis it has the form ${ }^{50-52}$

$$
2 b(t)=\mathbf{m} \cdot \mathbf{E}(t) .
$$


$\mathbf{m}=|\mathbf{m}| \mathbf{i}$, where $\mathbf{i}$ is a unit vector pointing in the direction of the external field and $|\mathbf{m}|=|e\langle 0+|\mathrm{r}| 0-\rangle|$ is the magnitude of the transition dipole moment associated with the tunnel doublet.

Our strategy will be to transform the Hamiltonian to a form suitable for a perturbation theory that is still valid when $\bar{V}$, the Franck-Condon renormalized tunnel splitting, is no longer a suitable expansion parameter. This is the case, for example, when $\bar{V}$ is large compared with the temperature or with the asymmetry between the initial and final states. $^{5,47,48,53,54}$ A projection operator method can be used to obtain an equation of motion for the system's density matrix to second order in the perturbation, that we will refer to as a kinetic equation. Previously, we carried out a projection operator analysis along these lines in the absence of an external field. ${ }^{47,48}$ When there is a time-dependent external field, a similar methodology can be developed, as we now show. Applying the unitary transformation

$$
U=\exp \left(\frac{i}{\hbar} s_{x} \sum_{j} \frac{\gamma_{j} p_{j}}{\omega_{j}^{2}}\right)
$$

to the Hamiltonian of Eq. (2.1), and dividing the interaction term in the transformed Hamiltonian into its thermally averaged part and the fluctuations about it leads to

$$
H=H_{\text {medium }}+H_{S}+H_{F}(t)+H_{\text {in }},
$$

where

$$
\begin{gathered}
H_{\text {medium }}=\sum_{j}\left(\frac{p_{j}^{2}}{2}+\omega_{j}^{2} \frac{q_{j}^{2}}{2}\right), \quad H_{S}=\bar{V} s_{z}, \\
H_{F}(t)=2 b(t) s_{x}, \quad H_{\mathrm{in}}=\frac{V}{2}\left(\Phi^{+} s_{z}+\Phi^{-} i s_{y}\right) .
\end{gathered}
$$

The interaction part of the Hamiltonian involves the operators $\Phi^{ \pm}$,

$$
\Phi^{+}=\Pi_{-}+\Pi_{+}-2\langle\Pi\rangle, \quad \Phi^{-}=\Pi_{-}-\Pi_{+},
$$

that are related to shifting operators $\Pi_{ \pm}$by

$$
\Pi_{ \pm}=\exp \left( \pm \frac{2 i}{\hbar} \sum_{j} \frac{\gamma_{j}}{\omega_{j}^{2}} p_{j}\right)
$$

and their equilibrium averages taken with $\rho_{b}$ $=\exp \left(-\beta H_{\text {medium }}\right) / \operatorname{tr}_{b} \exp \left(-\beta H_{\text {medium }}\right),{ }^{33}$ the density matrix describing the medium at equilibrium,

$$
\langle\Pi\rangle \equiv\left\langle\Pi_{ \pm}\right\rangle=\operatorname{tr}_{b} \rho_{b} \Pi_{ \pm}=\exp \left(-\frac{1}{\hbar} \sum_{j} \frac{\gamma_{j}^{2}}{\omega_{j}^{3}} \operatorname{coth}\left(\beta \hbar \omega_{j} / 2\right)\right),
$$

with $\beta=1 / k_{B} T$, and $\operatorname{tr}_{b}$ denoting a trace over the medium degrees of freedom. The quantity $\bar{V}$ in Eq. (2.4) is the Franck-Condon-renormalized tunnel splitting

$$
\bar{V}=V\langle\Pi\rangle \text {. }
$$

The Hamiltonian of Eq. (2.4) has been separated into several parts containing the system variable and its interaction with the external field, the medium variables, and a systemmedium interaction $H_{\text {in }}$. This latter term will be considered to be small.

The evolution of the overall density matrix $\rho(t)$ is given by the Liouville equation

$$
i \hbar \frac{\partial \rho}{\partial t}=[H(t), \rho(t)]
$$

We want a kinetic equation for $\sigma(t)=\operatorname{tr}_{b} \rho(t)$, the reduced density matrix of the system. To carry out this reduction, we use a projection operator method. ${ }^{55}$ A suitable projection operator $P$ is defined by $P O=\rho_{b} \operatorname{tr}_{b} O$. Applying this projection operator to Eq. (2.9) yields the exact but formal expression

$$
\begin{aligned}
& \frac{\partial \sigma(t)}{\partial t}=\operatorname{tr}_{b} L(t) \rho_{b} \sigma(t)+\int_{0}^{t} d \tau \operatorname{tr}_{b} L(t) \\
& \times\left(T e^{\int_{\tau}^{t} d s Q L(s) Q}\right) Q L(t) \rho_{b} \sigma(t)+\mathrm{IVT},
\end{aligned}
$$

where

$$
L(t)=L_{0}(t)+L_{1},
$$

$$
\begin{gathered}
L_{0}(t) \cdots \equiv-\frac{i}{\hbar}\left[H_{S}+H_{F}(t)+H_{\text {medium }}, \ldots\right] \\
\equiv L_{S}+L_{F}(t)+L_{b}, \\
L_{1} \cdots \equiv-\frac{i}{\hbar}\left[H_{\mathrm{in}}, \ldots\right],
\end{gathered}
$$

and $Q=1-P$. Here IVT denotes the contribution to the dynamics from the initial value of the density matrix. The choice of an initial ensemble $\rho(0)=\rho_{b} \sigma(0)$ makes the IVT zero. As the Hamiltonian has an explicit time dependence arising from the external field, we had to introduce a time ordering in Eq. (2.10) to deal with the noncommuting timedependent operators. ${ }^{56}$ We use the notation

$$
\begin{aligned}
T e^{\int_{\tau}^{t} d s Q L(s) Q} O \equiv & \left(T_{+} e^{-(i / \hbar) \int_{\tau}^{t} d s Q H(s) Q}\right) \\
& \times O\left(T_{-} e^{+(i / \hbar) \int_{\tau}^{t} d s Q H(s) Q}\right),
\end{aligned}
$$

where $T_{+}\left(T_{-}\right)$denotes time ordering with the largest time to the left (right).

Working to second order in $H_{\text {in }}$, and taking into account useful relations valid for any operator $A$,

$$
\begin{gathered}
P L_{0}(t) Q A=0, \quad Q L_{0}(t) Q A=L_{0}(t) Q A, \\
P L_{b} P Q=0, \\
P L_{1} Q A=P L_{1} A,
\end{gathered}
$$

leads to 


$$
\begin{aligned}
\frac{\partial \sigma(t)}{\partial t}= & -\frac{i}{\hbar}\left[H_{S}+H_{F}(t), \sigma(t)\right] \\
& -\frac{1}{\hbar^{2}} \int_{0}^{t} d \tau \operatorname{tr}_{b}\left[H_{\mathrm{in}},\left[\tilde{H}_{\mathrm{in}}(t, \tau), \rho_{b} \tilde{\sigma}(t, \tau)\right]\right],
\end{aligned}
$$

with the definitions

$$
\begin{aligned}
\tilde{H}_{\text {in }}(t, \tau)= & \left(T_{+} e^{-(i / \hbar) \int_{\tau}^{t} d s\left[H_{S}+H_{F}(s)+H_{b}\right]}\right) \\
& \times H_{\text {in }}\left(T_{-} e^{+(i / \hbar) \int_{\tau}^{t} d s\left[H_{S}+H_{F}(s)+H_{b}\right]}\right)
\end{aligned}
$$

and

$$
\begin{aligned}
\tilde{\sigma}(t, \tau)= & \left(T_{+} e^{-(i / \hbar) \int_{\tau}^{t} d s\left[H_{S}+H_{F}(s)\right]}\right) \\
& \times \sigma(\tau)\left(T_{-} e^{+(i / \hbar) \int_{\tau}^{t} d s\left[H_{S}+H_{F}(s)\right]}\right) .
\end{aligned}
$$

Use has been made of $\left[H_{S}, H_{b}\right]=\left[H_{F}(t), H_{b}\right]=0$, to simplify Eq. (2.15).

The difficulty in analyzing the above equation of motion centers on the time-ordering requirement arising from the noncommutativity of the time-dependent external field operator and the system operator $\left[H_{F}(t), H_{S}\right] \neq 0$. Specializing to the specific form of our Hamiltonian will permit progress, as evaluating this commutator with the use of Eq. (2.4b) shows that it is proportional to the small quantity $\bar{V} b(t)$. The modification of the evolution in Eqs. (2.14) and (2.15) from assuming commutativity is therefore negligible. Then Eq. (2.14) becomes

$$
\begin{aligned}
\tilde{\tilde{H}}_{\mathrm{in}}(t, \tau)= & \left(e^{-(i / \hbar)(t-\tau) H_{S}}\right)\left(e^{-(i / \hbar) \int_{\tau}^{t} d s H_{F}(s)}\right) \\
& \times\left(e^{-(i / \hbar)(t-\tau) H_{b}}\right) H_{\mathrm{in}}\left(e^{+(i / \hbar)(t-\tau) H_{b}}\right) \\
& \times\left(e^{+(i / \hbar) \int_{\tau}^{t} d s H_{F}(s)}\right)\left(e^{+(i / \hbar)(t-\tau) H_{S}}\right),
\end{aligned}
$$

and similarly for Eq. (2.15). With the form of $H_{\text {in }}$ in Eq. $(2.4 \mathrm{~b})$, we have

$$
\begin{aligned}
\left(e^{-(i / \hbar)(t-\tau) H_{b}}\right) H_{\mathrm{in}}\left(e^{+(i / \hbar)(t-\tau) H_{b}}\right) & \\
= & \frac{V}{2}\left(\Phi^{+}(\tau-t) s_{z}+\Phi^{-}(\tau-t) i s_{y}\right),
\end{aligned}
$$

where we have explicitly written the time dependence of the medium operators $\Phi^{ \pm}$in the Heisenberg representation. Note that the argument of the operators in Eq. (2.17) is $(\tau-t)$ according to the conventional relation between the operators in the Schrödinger and Heisenberg representations. ${ }^{33}$ The action of the field propagator on the spin operators in Eq. (2.16),

$$
\left(e^{-(i / \hbar) \lambda(t, \tau) s_{x}}\right) s_{\alpha}\left(e^{+(i / \hbar) \lambda(t, \tau) s_{x}}\right)=s_{\alpha}(\lambda(t, \tau)),
$$

where we have defined

$$
\lambda(t, \tau)=\int_{\tau}^{t} 2 b(s) d s
$$

which is readily found by generating second-order differential equations in $\lambda$ for the spin operators $s_{\alpha}(\lambda)$. This yields

$$
\begin{aligned}
& s_{z}(\lambda(t, \tau))=\cos 2 \lambda(t, \tau) s_{z}-\sin 2 \lambda(t, \tau) s_{y}, \\
& s_{y}(\lambda(t, \tau))=\cos 2 \lambda(t, \tau) s_{y}+\sin 2 \lambda(t, \tau) s_{z} .
\end{aligned}
$$

At this point we may obtain a more explicit expression for Eq. (2.13) by taking its matrix elements in the basis of eigenstates of $H_{S}$, the system Hamiltonian. We focus attention on the relaxation term, as the oscillating term's evaluation is straightforward. By expanding the double commutator and using the cyclic permutation property of the trace over the medium operators to rearrange terms, we ultimately find the kinetic equation

$$
\begin{aligned}
\frac{d \sigma_{i j}(t)}{d t}= & \left(-\frac{i}{\hbar}\left[\left(H_{S}+H_{F}(t)\right), \sigma(t)\right]\right)_{i j} \\
& +\sum_{k l} \int_{0}^{t} R_{i j k l}(t, \tau) \tilde{\tilde{\sigma}}_{k l}(t, \tau) d \tau,
\end{aligned}
$$

with

$$
\begin{aligned}
R_{i j k l}(t, \tau)= & \Gamma_{l j i k}^{+}(t, \tau)+\Gamma_{l j i k}^{-}(t, \tau)-\delta_{l j} \sum_{r} \Gamma_{i r r k}^{+}(t, \tau) \\
& -\delta_{i k} \sum_{r} \Gamma_{l r r j}^{-}(t, \tau), \\
\Gamma_{l j i k}^{+}(t, \tau)= & \frac{1}{\hbar^{2}} e^{-i \omega_{i k}(t-\tau)}\left\langle\widetilde{H}_{\mathrm{in}}(t-\tau)_{l j} \bar{H}_{\mathrm{in}}(\lambda(t, \tau))_{i k}\right\rangle, \\
\Gamma_{l j i k}^{-}(t, \tau)= & \frac{1}{\hbar^{2}} e^{-i \omega_{l j}(t-\tau)}\left\langle\bar{H}_{\mathrm{in}}(\lambda(t, \tau))_{l j} \widetilde{H}_{\mathrm{in}}(t-\tau)_{i k}\right\rangle,
\end{aligned}
$$

and we have defined $\bar{H}_{\text {in }}$ and $\widetilde{H}_{\text {in }}$ as

$$
\begin{gathered}
\bar{H}_{\mathrm{in}}(\lambda(t, \tau))_{l j}=\frac{V}{2}\left[\Phi^{+} s_{z}(\lambda(t, \tau))_{l j}+\Phi^{-} i s_{y}(\lambda(t, \tau))_{l j}\right] \\
\widetilde{H}_{\mathrm{in}}(t-\tau)_{l j}=\frac{V}{2}\left[\Phi^{+}(t-\tau)\left(s_{z}\right)_{l j}+\Phi^{-}(t-\tau)\left(i s_{y}\right)_{l j}\right]
\end{gathered}
$$

Recall that the brackets denote the averages over the equilibrium medium density matrix as given in Eq. (2.7). These equilibrium time correlation functions are nonstationary and therefore depend explicitly on $t$ and $\tau$ (not just their difference) because of the time dependence of the external field. $\widetilde{H}_{\text {in }}(t-\tau)$ represents the conventional Heisenberg operator at time $t-\tau$ obtained from the corresponding Schrödinger operator with the action of the medium propagator, while $\bar{H}_{\text {in }}(\lambda(t, \tau))$ denotes the operator obtained from the action of the external field propagator [cf. Eq. (2.18)].

The relaxation part of Eq. (2.20) is expressed in terms of the quantity $\tilde{\tilde{\sigma}}(t, \tau)$ that we now need to express in terms of $\sigma(\tau)$ in order to obtain a closed equation for $\sigma$. These reduced density matrices are connected via Eq. (2.15). Thus the relaxation equation in Eq. (2.20) can be written as 


$$
\begin{aligned}
\frac{d \sigma_{i j}(t)}{d t}= & \left(-\frac{i}{\hbar}\left[\left(H_{S}+H_{F}(t)\right), \sigma(t)\right]\right)_{i j} \\
& +\sum_{k l} \int_{0}^{t} \widetilde{R}_{i j k l}(t, \tau) \sigma_{k l}(\tau) d \tau,
\end{aligned}
$$

where the tilde on $\widetilde{R}_{i j k l}$ symbolizes the redefined relaxation tensor obtained from the conversion of the $\tilde{\tilde{\sigma}}(t, \tau)$ matrix elements to the $\sigma(\tau)$ matrix elements. We stress that Eqs. (2.20) and (2.22) are just different expressions of the same kinetic equation.

The kinetic equation as written in Eq. (2.22) is not of the convolution structure because of the presence of the timedependent external field. If the external field were constant (or absent), then Eq. (2.22) would have a convolution structure and could be expressed in Laplace transform space with the transformed relaxation kernel $\widehat{\widetilde{R}}(\mu)$. (The caret denotes the Laplace transform of the function, and we use $\mu$ for the Laplace transform argument.) The conventional time local kinetic equation is obtained by replacing $\widehat{\widetilde{R}}(\mu)$ with $\widehat{\widetilde{R}}(\mu$ $=0)$. This replacement is correct when $\widehat{\widetilde{R}}(\mu)$ is an analytic function of $\mu$ in the neighborhood of $\mu=0$. A case where this replacement is not valid is for very low temperatures and a medium whose mode spectrum [the $\omega_{j}$ 's of Eq. (2.1)] is Ohmic. Here $\widetilde{\widetilde{R}}(\mu)$ has a branch point at $\mu=0$. The consequences of this feature have been discussed in detail by Leggett et al. ${ }^{8}$ Even in the Ohmic case, when the temperature is not so low, time-local equations give a satisfactory account of the dynamics. ${ }^{34}$ In the following we will assume the adequacy of this replacement, as it describes the kinetics in most regimes.

A connection with a local-in-time, Redfield-like theory, ${ }^{57}$ including the effect of an external field, can now be made. Equation (2.15) shows that another way of writing $\tilde{\sigma}(t, \tau)$ is as $\sigma^{0}(t)$, where this latter notation expresses the feature that $\sigma^{0}(t)$ is the zero-order solution of Eq. (2.20a) with the condition that at the initial time $t=\tau$, the density matrix was $\sigma(\tau)$. Therefore, in Eq. (2.20a), $\tilde{\sigma}(t, \tau)$ can be replaced by $\sigma^{0}(t)$. Since Eq. (2.20) has been obtained by second-order perturbation theory, it is correct, to the order of the calculation, to replace $\sigma^{0}(t)$ by $\sigma(t)$. As a consequence, we obtain the following Redfield-like kinetic equation:

$$
\begin{aligned}
\frac{d \sigma_{i j}(t)}{d t}= & \left(-\frac{i}{\hbar}\left[\left(H_{S}+H_{F}(t)\right), \sigma(t)\right]\right)_{i j} \\
& +\sum_{k l}\left[\int_{0}^{\infty} R_{i j k l}(t, \tau) d \tau\right] \sigma_{k l}(t) .
\end{aligned}
$$

We stress that, even if the kinetic equation in Eq. (2.22) is approximated by its time-local version, it still differs from Eq. (2.23) as the relaxation tensors are different. The difference arises from the presence of the external field. In the absence of the external field, the time evolution connecting $\tilde{\tilde{\sigma}}(t, \tau)$ and $\sigma(\tau)$ is slow, as it originates from the small quantity $\bar{V}$. Thus, without an external field, and assuming the existence of the time integral in Eq. (2.23) [or that $\widehat{\widetilde{R}}_{i j k l}(\mu$ $=0)$ exist], Eqs. (2.22) and (2.23) are equivalent. When the external field is present, the interaction representation evolu- tion in Eq. (2.15) includes its potentially significant effect on the system dynamics and leads to differences in the forms of the kinetic equations. Explicit equations expressing this difference are presented below.

The analysis of the population dynamics implied by the above kinetic equation requires solutions of the coupled equations of motions for the density-matrix elements. The equations of motion have coefficients given by $R_{i j k l}$, the elements of the relaxation tensor $R$. These elements can be expressed in terms of the medium, system-medium, and system-external field coupling parameters. This calculation is carried out in the Appendix. We first focus on the structure of the equations of motion, and then will list the various independent elements of $R$. Since a two-level system's density matrix is characterized by three independent quantities, we may choose the following linear combinations as our variables:

$$
\begin{gathered}
p(t)=\sigma_{12}(t)+\sigma_{21}(t), \\
d_{-}(t)=i\left(\sigma_{21}(t)-\sigma_{12}(t)\right), \\
d_{+}(t)=\sigma_{11}(t)-\sigma_{22}(t) .
\end{gathered}
$$

On the basis of eigenstates of the system Hamiltonian, $p(t)$ is the difference in population between the left and right localized states, the quantity of interest for tunneling dynamics.

We now analyze the general kinetic equation, Eq. (2.20). To do so it is convenient to relate the matrix elements of $\tilde{\sigma}(t, \tau)=\sigma^{0}(t)$ to those of $\sigma(\tau)$ according to the relation of Eq. (2.15), which is simply a rotation. Thus

$$
\left(\begin{array}{l}
p^{0}(t) \\
d_{+}^{0}(t) \\
d_{-}^{0}(t)
\end{array}\right)=\left(\begin{array}{ccc}
1 & 0 & 0 \\
0 & \cos 2 \lambda(t, \tau) & -\sin 2 \lambda(t, \tau) \\
0 & \sin 2 \lambda(t, \tau) & \cos 2 \lambda(t, \tau)
\end{array}\right)\left(\begin{array}{c}
p(\tau) \\
d_{+}(\tau) \\
d_{-}(\tau)
\end{array}\right) .
$$

With this connection, and Eq. (2.20), we eventually obtain the following equations of motion:

$$
\begin{aligned}
\dot{p}(t)= & 2(\bar{V} / \hbar) d_{-}(t)+\int_{0}^{t}[g(t, \tau)+c(t, \tau)] p(\tau) d \tau \\
& +\int_{0}^{t}[\alpha(t, \tau)+\beta(t, \tau)] d \tau, \\
\dot{d}_{-}(t)= & -2(\bar{V} / \hbar) p(t)+4(b(t) / \hbar) d_{+}(t) \\
& +\int_{0}^{t}\{[g(t, \tau)-c(t, \tau)] \cos (2 \lambda(t, \tau)) \\
& +i[\alpha(t, \tau)-\beta(t, \tau)] \sin (2 \lambda(t, \tau))\} d_{-}(\tau) d \tau \\
& +\int_{0}^{t}\{i[\beta(t, \tau)-\alpha(t, \tau)] \cos (2 \lambda(t, \tau)) \\
& +[g(t, \tau)-c(t, \tau)] \sin (2 \lambda(t, \tau))\} d_{+}(\tau) d \tau,
\end{aligned}
$$




$$
\begin{aligned}
\dot{d}_{+}(t)= & -4(b(t) / \hbar) d_{-}(t)+\int_{0}^{t}\{2 c(t, \tau) \cos (2 \lambda(t, \tau)) \\
& +2 i \gamma(t, \tau) \sin (2 \lambda(t, \tau))\} d_{+}(\tau) d \tau \\
& +\int_{0}^{t}\{2 i \gamma(t, \tau) \cos (2 \lambda(t, \tau)) \\
& -2 c(t, \tau) \sin (2 \lambda(t, \tau))\} d_{-}(\tau) d \tau .
\end{aligned}
$$

The coefficients in these equations are expressed in terms of the elements of the relaxation tensor $R$ as follows:

$$
\begin{gathered}
\alpha=R_{1211}, \quad \beta=R_{2111}, \quad g=R_{1212}, \quad c=R_{1111}, \\
\gamma=R_{1112} .
\end{gathered}
$$

The "true" Redfield equations would be obtained by replacing $\tilde{\sigma}(t, \tau)$ by $\sigma(t)$ in Eq. (2.20) and, as usual, by extending the integration limit to infinity. The resulting equations of motion for the two-level system are

$$
\begin{gathered}
\dot{p}(t)=2(\bar{V} / \hbar) d_{-}(t)+\int_{0}^{\infty}[g(t, \tau)+c(t, \tau)] d \tau p(t) \\
+\int_{0}^{\infty}[\alpha(t, \tau)+\beta(t, \tau)] d \tau \\
\dot{d}_{-}(t)=-2(\bar{V} / \hbar) p(t)+4(b(t) / \hbar) d_{+}(t) \\
+\int_{0}^{\infty}[g(t, \tau)-c(t, \tau)] d \tau d_{-}(t) \\
+i \int_{0}^{\infty}[\beta(t, \tau)-\alpha(t, \tau)] d \tau d+(t), \\
\dot{d}_{+}(t)=-4(b(t) / \hbar) d_{-}(t)+2 \int_{0}^{\infty} c(t, \tau) d \tau d_{+}(t) \\
+2 i \int_{0}^{\infty} \gamma(t, \tau) d \tau d_{-}(t) .
\end{gathered}
$$

Note that the equation for the population evolution $\dot{p}(t)$ has the same structure for both approaches but that the equations for the other elements differ. The differences are due to the external field term $\lambda(t, \tau)$ as noted above, in general. However, as we now show, approximate solutions of Eqs. (2.26) and (2.28) will coincide. So, in this sense, the projection operator and Redfield approaches can lead to the same predictions.

For our model Hamiltonian there are only five independent elements. Their explicit expressions are presented in the Appendix. Along with the definition of $\lambda(t, \tau)$ in Eq. (2.18b), they completely specify the equations of motion. Two combinations that will be relevant to approximate solutions of these equations are $\alpha+\beta$ and $c+g$. Their explicit forms, obtained with the use of Eq. (A6), are

$$
\begin{aligned}
g(t, \tau) & +c(t, \tau) \\
& =-2(\bar{V} / \hbar)^{2}\left[e^{W(t-\tau)}+e^{W(\tau-t)}-2\right] \cos 2 \lambda(t, \tau)
\end{aligned}
$$

and

$$
\alpha(t, \tau)+\beta(t, \tau)=2(\bar{V} / \hbar)^{2}\left[e^{W(\tau-t)}-e^{W(t-\tau)}\right] i \sin 2 \lambda(t, \tau),
$$

where $W(t)$ is defined in Eq. (A5). Note that Eq. (A5) relates $W(t)$ to the spectral density of the system-medium interaction.

Clearly, Eqs. (2.26) [or Eqs. (2.28)] are very complex. We first analyze them for a constant external field. For an initial condition $p(0)=1, d_{+}(0)=d_{-}(0)=0$, corresponding to the system localized on the left side, an accurate approximate equation of motion can be developed. Since the relaxation terms in the second and third equations in Eq. (2.26) are explicitly of order $\bar{V}^{2}$, and, considering that $d_{ \pm}(t)$ vanish initially, their effect on the evolution of $p(t)$ may be neglected except perhaps for very long times. Then, the equations of motion in Eq. (2.26) simplify to

$$
\begin{gathered}
\dot{p}(t)=2(\bar{V} / \hbar) d_{-}(t)+\int_{0}^{t}[g(t-\tau)+c(t-\tau)] p(\tau) d \tau \\
+\int_{0}^{t}[\alpha(t-\tau)+\beta(t-\tau)] d \tau \\
\dot{d}_{-}(t)=-2(\bar{V} / \hbar) p(t)+4(b / \hbar) d_{+}(t)+O\left(\bar{V}^{2}\right) \\
\dot{d}_{+}(t)=-4(b / \hbar) d_{-}(t)+O\left(\bar{V}^{2}\right) .
\end{gathered}
$$

If the Redfield Eqs. (2.28) were approximated in the same spirit, the equations of motion would be the local-in-time analog of Eqs. (2.31). Thus, to the extent that local equations are adequate, the Redfield and projection operator based results will coincide. Equations (2.31) do, however, have a convolution structure that will permit Laplace transformation. Formally solving the second and third equations for $d_{-}(t)$ and inserting the first equation provides a closed equation for $p(t)$, whose Laplace transformed solution is a generalization $^{48}$ of the so-called noninteracting blip approximation. ${ }^{8}$ The advantage of this convolution formulation is its validity at temperatures smaller than $\bar{V}$. The tunnel system's interaction with the medium at such low temperatures may not be sufficiently strong as to completely destroy the coherence of the tunneling event. There is no guarantee of a rate process, as would be implied by a time-local kinetic equation. As the temperature is raised, $\bar{V}$ decreases rapidly, and then incoherent tunneling, as described by a rate process, is obtained. In this regime, the inhomogeneous term in Eq. (2.31) accounts for the decay of $p(t)$ to its correct long-time equilibrium value ${ }^{8}$

$$
p_{\text {eq }}=-\tanh \left(2 b / k_{B} T\right) .
$$

This gives the correct Boltzmann population distribution of the left and right states (for $\bar{V}$ small compared to $b$ ).

\section{CONTROL OF TUNNELING AT LOW TEMPERATURES}

For a time-dependent external field, the nonconvolution structure of the above equations of motion precludes a simple analysis in terms of Laplace transforms for arbitrary time variations. Nevertheless, we should anticipate that, 
while a rate regime is strictly precluded here, due to the external field's time dependence, a rate regime should emerge as a suitable average of a time-dependent relaxation quantity. In particular, for a sinusoidal variation of the external field, a cycle-averaged rate constant can be expected to describe the population decay. We can proceed analytically by again using the physically interesting initial condition $p(0)=1, d_{+}(0)=d_{-}(0)=0$. Also, we shall assume that the renormalized tunnel splitting $\bar{V}$ is a negligible quantity, even though $V=\bar{V} /\langle\Pi\rangle=\bar{V} e^{W(0) / 2}$ is itself small but not negligible. That $\bar{V}$ is much smaller than $V$ is a well-known feature of a fermionic bath, as characterized by an Ohmic spectrum. ${ }^{58}$ The temperature then will also be assumed to be large compared with $\bar{V}$, so that the tunneling is in the incoherent regime. In the studies of defect tunneling in metals noted in Sec. I, where an Ohmic spectrum is assumed to characterize the coupling between the defect and the conduction electrons, the $V$ values found are many orders of magnitude smaller than the temperature regime $(0.1-2.0 \mathrm{~K})$ of the experiment. ${ }^{35-37}$ Thus a rate regime is the appropriate one to focus on. Even for, in this sense, "high" temperatures, the medium dynamics may still have to be treated quantum mechanically, as $\beta \hbar \omega$ may not be small. Under these conditions, terms proportional to $\bar{V}$ and $\bar{V}^{2}$ are neglected, while those proportional to $V^{2}$ are kept in Eqs. (2.26). In this way the evolution of $p(t)$ is decoupled from that of $d_{ \pm}(t)$, and is given by the first order in time equation

$$
\dot{p}(t)=-\int_{0}^{t}[k(t, \tau)] p(\tau) d \tau+\int_{0}^{t}[\alpha(t, \tau)+\beta(t, \tau)] d \tau,
$$

where the rate kernel $k(t, \tau)$ is defined as

$$
\begin{aligned}
k(t, \tau) & \equiv-(g(t, \tau)+c(t, \tau)) \\
& =2(\bar{V} / \hbar)^{2}\left[e^{W(t-\tau)}+e^{W(\tau-t)}-2\right] \cos 2 \lambda(t, \tau) \\
& \approx 2(V / \hbar)^{2}\left[e^{W(t-\tau)-W(0)}+e^{W(\tau-t)-W(0)}\right] \cos 2 \lambda(t, \tau) \\
& =(2 V / \hbar)^{2} e^{\Psi(t-\tau)-\Psi(0)} \cos G(t-\tau) \cos 2 \lambda(t, \tau) .
\end{aligned}
$$

The rate kernel $k(t, \tau)$ is the sum of the forward $k_{f}(t, \tau)$ and backward $k_{b}(t, \tau)$ (left to right and right to left) rate kernels. They are readily obtained as

$$
\begin{aligned}
k(t, \tau)= & k_{f}(t, \tau)+k_{b}(t, \tau) \\
= & \frac{1}{2}(2 V / \hbar)^{2} e^{\Psi(t-\tau)-\Psi(0)}\{\cos [G(t-\tau)-2 \lambda(t, \tau)] \\
& +\cos [G(t-\tau)+2 \lambda(t, \tau)]\} .
\end{aligned}
$$

At high temperature $\beta \hbar \omega \ll 1$, the forward rate kernel obtained from Eq. (3.3) coincides with the result we obtained by using a classical bath analysis at the outset. ${ }^{13}$ In the quantum regime, rate equations similar to Eq. (3.1) have recently been obtained by other methods. ${ }^{21,22,26-29}$

We now analyze the effects arising from a quantum medium in the presence of a time-dependent external field on the quantity

$$
\Gamma(t)=\int_{0}^{\infty} k(t, \tau) d \tau
$$

that we will refer to as the time-dependent rate, and the corresponding quantities $\Gamma_{f}(t)$ and $\Gamma_{b}(t)$ for the forward and backward rates. If a rate regime is to emerge, we must assume that the kernel $k(t, \tau)$ decays rapidly relative to the population decay, and that this time scale separation leads to the relaxation equation

$$
\dot{p}(t)=-\Gamma(t) p(t)+\int_{0}^{t}[\alpha(t, \tau)+\beta(t, \tau)] d \tau .
$$

For a sinusoidal external field whose variation is rapid relative to the population decay, it is appropriate to introduce averaged rate constants $\Gamma_{\mathrm{av}}, \Gamma_{f, \mathrm{av}}$, and $\Gamma_{b, \text { av }}$ according to, e.g.,

$$
\Gamma_{\mathrm{av}}=\frac{\Omega}{2 \pi} \int_{0}^{2 \pi / \Omega} \Gamma(t) d \tau
$$

While the experimental quantity of greatest interest and accessibility is the averaged rate constant, we will first analyze the time-dependence of, e.g., $\Gamma_{f}(t)$, to see under what conditions it, too, is roughly constant.

The quantity $\Gamma_{f}(t)$ can be written in terms of the spectral density $J(\omega)$ by use of Eqs. (A5) and (3.3) in Eq. (3.4):

$$
\Gamma_{f}(t)=\frac{1}{2}(2 V / \hbar)^{2} \int_{0}^{\infty} e^{W(\tau)-W(0)} e^{i \varepsilon \tau} e^{i 2 \lambda(t, \tau)} d \tau,
$$

with

$$
\begin{aligned}
W(t)-W(0)= & \Psi(t)-\Psi(0)-i G(t) \\
= & \int_{\omega_{\min }}^{\omega_{\max }}\left(J(\omega) / \omega^{2}\right)[\operatorname{coth}(\beta \hbar \omega / 2)(\cos \omega t-1) \\
& -i \sin \omega t] d \omega .
\end{aligned}
$$

In the absence of an external field, this is the conventional expression for the rate coefficient for tunneling in a two-level system of asymmetry $\varepsilon$ coupled to a bath whose couplings are described by $J(\omega){ }^{1,5,8}$ To be specific, we now introduce the Ohmic spectrum $J(\omega)=2 \alpha \omega$ for $0<\omega<\omega_{c}$, where $\alpha$ is the coupling strength $\left(\alpha<\frac{1}{2}\right)$, and $\omega_{c}$ is a cutoff frequency whose energy is much larger than the thermal energy and the tunnel splitting. As noted in Sec. I, this spectral density can characterize the interaction of a tunneling defect with the conduction electrons of a metal. ${ }^{35-37}$ For the Ohmic spectral density, ${ }^{38}$

$$
\begin{aligned}
W(t)-W(0)= & -2 \alpha \ln \left[\sqrt{1+\left(\omega_{c} t\right)^{2}} \frac{\sinh \pi k_{B} T t / \hbar}{\pi k_{B} T t / \hbar}\right] \\
& -i 2 \alpha \tan ^{-1}\left(\omega_{c} t\right) .
\end{aligned}
$$

It is convenient to use the external field

$$
b(t)=b_{c}+b \cos \Omega t,
$$

as it readily yields results for a constant and a sinusoidal external field. With the definition of $\lambda(t, \tau)$ in Eq. (2.18b), $\Gamma_{f}(t)$ then is given as 

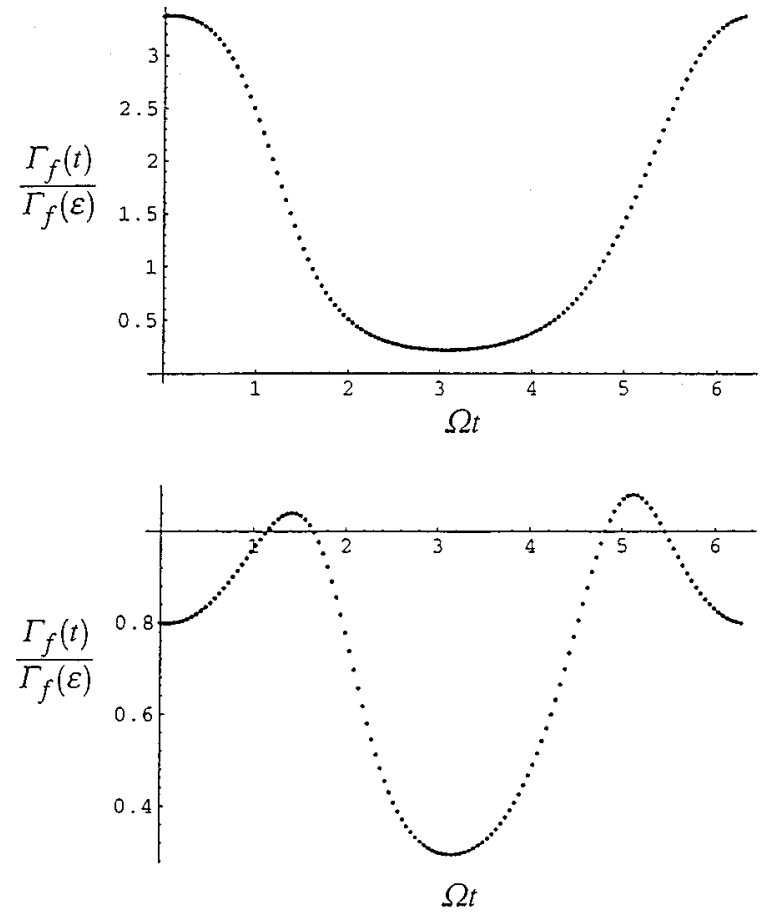

FIG. 1. The time-dependent rate $\Gamma_{f}(t)$ relative to the field-free rate constant $\Gamma_{f}(\varepsilon)$ for one cycle of the external field. The parameters (in K) are $\varepsilon=-1$ (top panel) and $\varepsilon=0$ (bottom panel); $4 b=1.0$ and $\Omega=0.1$.

$$
\begin{aligned}
\Gamma_{f}(t)= & \frac{1}{2}(2 V / \hbar)^{2} \operatorname{Re} \int_{0}^{\infty} e^{W(\tau)-W(0)} e^{i\left(\varepsilon+4 b_{c}\right) \tau} \\
& \times e^{i(4 b / \hbar \Omega)[\sin (\Omega t)-\sin (\Omega t-\Omega \tau)]} d \tau .
\end{aligned}
$$

For a constant external field $b=0$, this expression shows that the asymmetry of the rate constant has been changed from $\varepsilon$ to $\varepsilon+b_{c}$. This shift can, of course, modify the rate constant dramatically for appropriate choices of these parameters. The effects of a time-dependent external field are obtained by numerical integration of Eq. (3.11) with Eq. (3.9). As long as the cutoff is suitably large, the results are independent of its value. We present $\Gamma_{f}(t)$ normalized by the value in the absence of the external field, $\Gamma_{f}(\varepsilon)$, as defined by Eq. (3.18) below. We set $b_{c}=0$, since any asymmetry can be expressed directly through $\varepsilon$, even though the source is different, of course. It is clear, from the explicit form of Eqs. (3.11) and (3.9), that the dimensionless quantities

$$
\varepsilon / 2 \pi k_{B} T, \quad \hbar \Omega / 2 \pi k_{B} T, \quad 4 b / \hbar \Omega
$$

are appropriate units of measurement, and we will use the corresponding temperatures for $\varepsilon, \Omega$, and $4 b$ in listing the parameter values. The results are presented for a selection of the asymmetries $\varepsilon=-1,0$, and 1 corresponding to endothermic (uphill), symmetric, and exothermic (downhill) reactions, in the forward direction. We have used $\alpha=0.216$, as this is one value found in the experiments and also makes $2 \pi k_{B} T \alpha=1.0 \mathrm{~K}$ for $T=1 \mathrm{~K}$. The field strength $4 b$ is kept at $1 \mathrm{~K}$, and $\Omega$ is varied from 0.1 to $10 \mathrm{~K}\left(1 \mathrm{~K}=1.3 \times 10^{11} \mathrm{~s}^{-1}\right)$ to span external frequencies that are small to large as compared with $2 \pi k_{B} T \alpha$ at $1 \mathrm{~K}$. Figures $1-3$, summarizing these

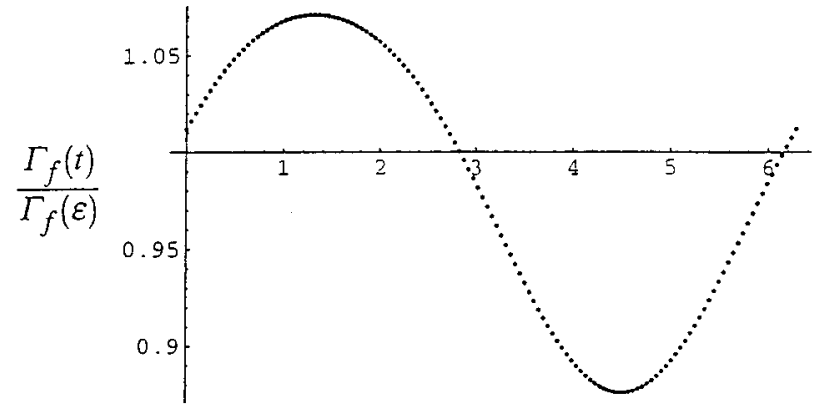

$\Omega t$

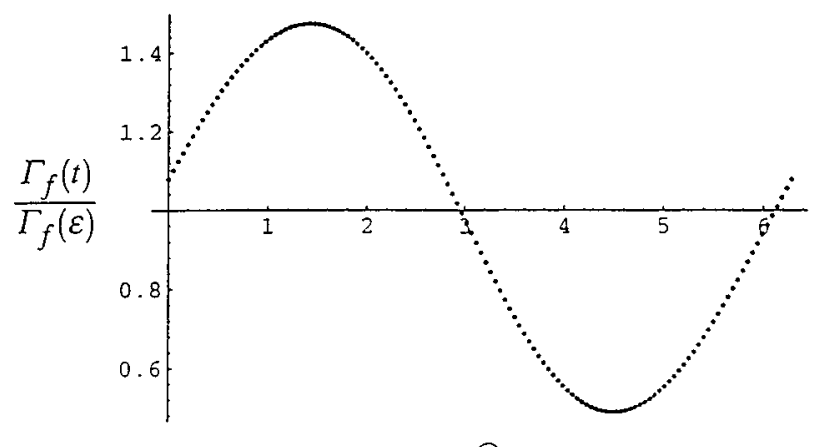

$\Omega t$

FIG. 2. The time-dependent rate $\Gamma_{f}(t)$ relative to the field-free rate constant $\Gamma_{f}(\varepsilon)$ for one cycle of the external field. The parameters (in K) are $\varepsilon=-1$ (top panel) and $\varepsilon=0$ (bottom panel); $4 b=1.0$ and $\Omega=5.0$.

results, are plotted in units of $\Omega t$. Figure 1 , where $\Omega$ is small $\left(\Omega / 2 \pi k_{B} T \alpha=0.1\right)$, shows that $\Gamma_{f}(t)$ has a substantial variation over its period. The cosine symmetry is readily obtained by expanding $\sin \Omega \tau$ as $\Omega \tau$ in Eq. (3.11) to obtain the approximate express

ion

$$
\Gamma_{f}(t)=\frac{1}{2}(2 V / \hbar)^{2} \operatorname{Re} \int_{0}^{\infty} e^{W(\tau)-W(0)} e^{i[\varepsilon+(4 b / \hbar) \cos (\Omega t)] \tau} d \tau
$$

This shows that a time-dependent asymmetry can be defined as $\varepsilon(t)=\varepsilon+(4 b / \hbar) \cos (\Omega t)$, and Eq. (3.12) for $\Gamma_{f}(t)$ can be integrated ${ }^{8}$ to yield

$$
\begin{aligned}
\Gamma_{f}(t)= & \left(V_{r} / \hbar\right)\left[\frac{2 \pi k_{B} T}{V_{r}}\right]^{2 \alpha-1} \exp \left(\varepsilon(t) / 2 k_{B} T\right) \frac{\sin \pi \alpha}{2 \pi} \\
& \times\left|\Gamma\left(\alpha+\frac{i \varepsilon(t)}{2 \pi k_{B} T}\right)\right|^{2},
\end{aligned}
$$

where $\Gamma(z)$ is the gamma function of complex argument $z$, and $V_{r}=V\left(V / \omega_{c}\right)^{\alpha / 1-\alpha}$. The parameter $V_{r}$ is the renormalized tunnel splitting.

At the other extreme of large $\Omega$, the oscillations of $\sin (\Omega t$ $-\Omega \tau$ ) should average to zero. Then Eq. (3.11) can be reduced to

$$
\Gamma_{f}(t)=\frac{1}{2}(2 V / \hbar)^{2} \operatorname{Re} \int_{0}^{\infty} e^{W(\tau)-W(0)} e^{i \varepsilon \tau} d \tau e^{i(4 b / \hbar \Omega) \sin (\Omega t)}
$$



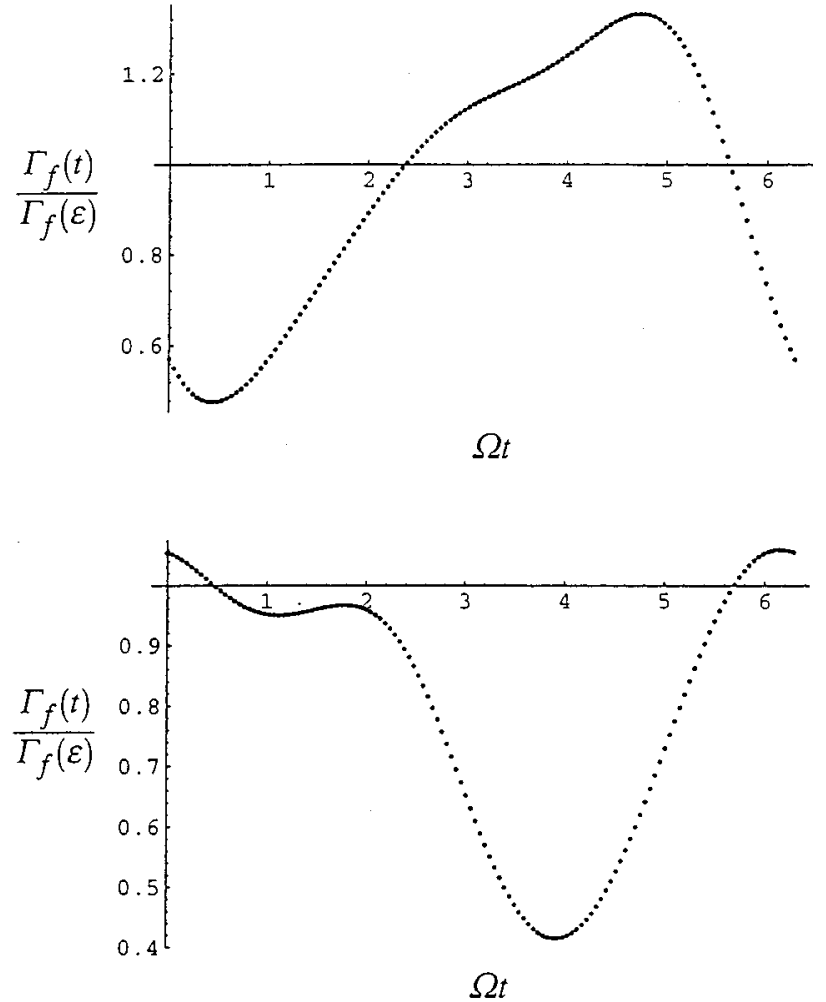

FIG. 3. The time-dependent forward rate $\Gamma_{f}(t)$ relative to the field-free rate constant $\Gamma_{f}(\varepsilon)$ for one cycle of the external field. The parameters (in K) are $\varepsilon=-1$ (top panel) and $\varepsilon=0$ (bottom panel); $4 b=1.0$ and $\Omega=1.0$.

If in addition $4 b / \hbar \Omega<1$, we may expand in this factor to obtain, schematically,

$$
\Gamma_{f}(t) / \Gamma_{f}(\varepsilon)=1+A(\varepsilon) \frac{4 b}{\hbar \Omega} \sin (\Omega t),
$$

where $A(\varepsilon)$ is a quotient of integrals involving the spectral density and depends significantly on the value of $\varepsilon$. Figure 2 shows the oscillation of Eq. (3.15). Note, too, that the averaged rate constant $\Gamma_{f \text {,av }}$ is predicted to be one from Eq. (3.15). This follows from the assumed small value of $4 b / \hbar \Omega$, as we shall see below. For large values of $\Omega / 2 \pi \alpha k_{B} T$ but $4 b / \hbar \Omega$ not small, the oscillation is not well described by Eq. (3.15) (it is not of symmetric magnitude around unity) but the magnitude of the oscillations is not very large. For intermediate values of $\Omega / 2 \pi \alpha k_{B} T$, we have not been able to obtain a simple expression to characterize the oscillations shown in Fig. 3. The variation over a cycle is about a factor of 2. Thus we may anticipate that an averaged formula should be useful, the more so as $\Omega / 2 \pi \alpha k_{B} T$ increases.

A formula for the averaged rate constant $\Gamma_{f \text {,av }}$ can be obtained by use of the expansion ${ }^{23}$

$$
e^{i(4 b / \hbar \Omega) \sin \Omega t}=\sum_{p=-\infty}^{p=\infty} J_{p}(4 b / \hbar \Omega) e^{i p \Omega t} .
$$

Its use in Eq. (3.9) with Eq. (3.11) permits Eq. (3.6) to be reduced to

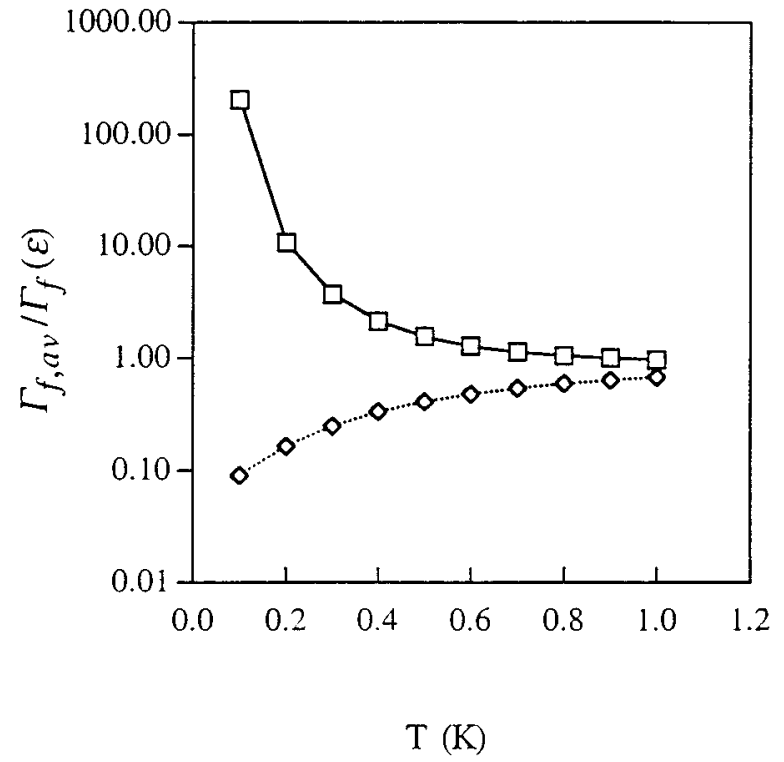

FIG. 4. The averaged forward rate constant $\Gamma_{f \text {,av }}$ relative to the field-free rate constant $\Gamma_{f}(\varepsilon)$ as a function of the temperature. A time-dependent external field with $4 b / \hbar \Omega=1.202$ and $\varepsilon=-\Omega=$ $-0.5 \mathrm{~K}$ for the solid line and $\varepsilon=0$ with $\Omega=0.5 \mathrm{~K}$ for the dotted line.

$$
\Gamma_{f, \mathrm{av}}=\sum_{m=-\infty}^{m=-\infty} J_{m}^{2}\left(\frac{4 b}{\hbar \Omega}\right) \Gamma_{f}\left(\varepsilon+4 b_{c}+m \Omega\right)
$$

where we have defined

$$
\Gamma_{f}(c)=\frac{1}{2}(2 V / \hbar)^{2} \operatorname{Re} \int_{0}^{\infty} e^{W(\tau)-W(0)} e^{i c \tau} d \tau .
$$

Thus, for a time-dependent external field with $b_{c}=0$, the averaged rate constant is a Bessel-function-weighted sum of rate constants of asymmetries $(\varepsilon+m \Omega)$. This suggests the following control of tunneling strategies. ${ }^{23-25,28}$ To make the averaged rate constant large, relative to the field-free rate constant, arrange matters to minimize the $m=0$ rate constant and/or Bessel-function term in the sum in Eq. (3.17). For example, if we choose $4 b / \hbar \Omega=2.4048 \ldots$, the first zero of the $J_{0}$ Bessel function, and take $\varepsilon=\Omega$ such that the rate constant $\Gamma_{f}(\varepsilon)$ will be small relative to $\Gamma_{f}(\varepsilon-\Omega)$, then the external field will enhance the averaged rate constant. Figure 4 illustrates this feature for the parameters indicated in the figure legend. That the difference in rates increases at lower temperature reflects the increasing value of $\varepsilon / k_{B} T$. To decrease the rate relative to the field-free case can be accomplished by again eliminating the $m=0$ term in Eq. (3.17), but now enhancing $\Gamma_{f}(\varepsilon)$ relative to $\Gamma_{f}(\varepsilon \pm \Omega)$. Figure 4 displays this external-field-produced rate reduction.

Finally, as noted above, a constant external field acts simply as an additional source of asymmetry. Thus appropriate choices of a constant external field should also be effective in modulating the tunnel rate. An increase and decrease relative to the no external field rate are shown in Fig. 5. 


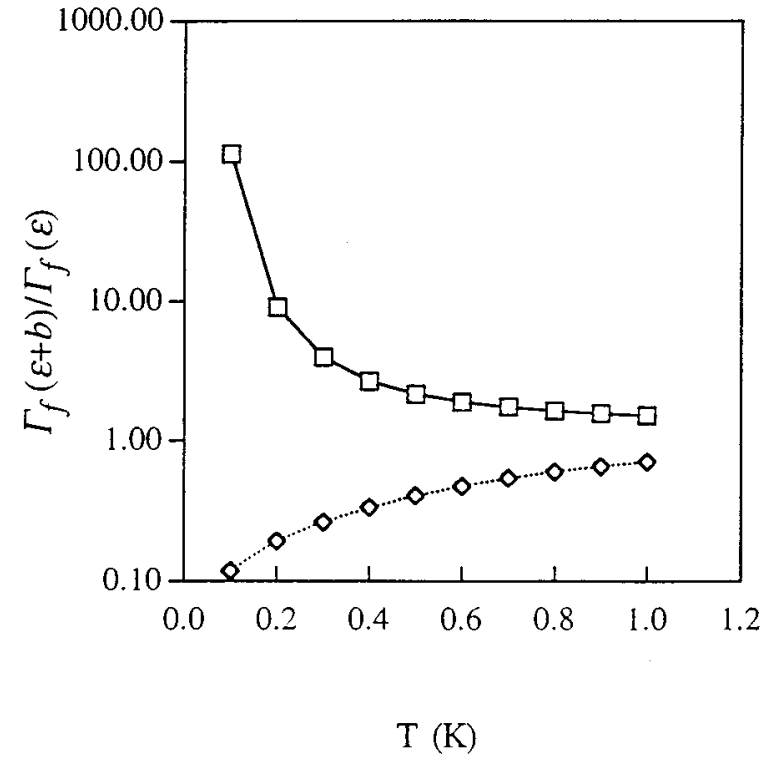

FIG. 5. The forward rate constant $\Gamma_{f}(\varepsilon+b)$ relative to the fieldfree rate constant $\Gamma_{f}(\varepsilon)$ as a function of the temperature. Here $4 b_{c}=1.2024 \mathrm{~K}$ and $\varepsilon=0.5 \mathrm{~K}$ for the solid line and $\varepsilon=0$ for the dotted line.

\section{CONCLUDING REMARKS}

The projection operator method that we have used has provided the equations of motion of Eq. (2.20). The expansion is carried out in terms of $H_{\text {in }}$ [cf. Eq. (2.4)], whose thermal average is zero. The rate equations that are extracted from the equations of motion are valid when $\beta \hbar \bar{V}$ is small, as we use an approximation scheme appropriate for a rate regime. This corresponds ${ }^{47}$ to the so-called noninteracting blip approximation ${ }^{8}$ (NIBA), in the absence of the external field. The inclusion of the external field should not invalidate this approximation scheme, as its effect tends to decrease the size of the dissipative terms that contribute to the perturbation [cf. Eqs. (A1)]. Furthermore, as the external field does not appear in $\bar{V}$, the assumed smallness of $\bar{V}$ is not affected by the external field. It is possible to extend the analysis to the regime of lower temperature in the sense that $\beta \hbar \bar{V}$ is not small, as we did previously for nondriven systems, by choosing an appropriate projection operator. ${ }^{48}$ For $T=0$, recent work by Chakravarty and Rudnick ${ }^{59}$ cast some doubt on the validity of NIBA-type solutions to describe the dynamics of the equilibrium time-correlation function in the sense that its time behavior is different than that of the population, $p(t)$. As we are concerned with finite temperature, and our focus is on the population's time evolution, in contrast to the correlation function, such problems do not arise here.

A difficulty that arises from the use of a time-dependent external field is the lack of commutativity of this term with the system Hamiltonian. However, as noted above Eq. (2.16), the neglect of this noncommutativity should only introduce a small error. Then, as long as the interaction Hamiltonian can be written as a product of system and medium operators, the structure in Eq. (2.20) will be obtained. In particular, the kinetic equation applies to externally driven systems with several tunnel doublets. Of interest in this case would be the possibility of externally induced transitions between different tunnel doublets and their effect on the population transfer from one localized state to the other.

An interesting consequence of a time-dependent external field is the different equations of motion that result from the projection operator and "Redfield" procedures. This is, to our knowledge, a new effect, in addition to the conventional one, whereby the Redfield methodology leads to time-local equations, while the projection operator method leads to a convolution structure that can accommodate a nonrate regime, even in the absence of a time-dependent external field. The fast variation of the external field, present in the interaction representation of Eq. (2.15), is responsible for this difference in the equations of motion.

The solution of the equations of motion that we obtained in Sec. II relies on the feature that the Franck-Condon renormalized splitting $\bar{V}$ is small compared with the bare $V$. Then the three equations of motion, Eqs. (2.26), decouple to provide a population evolution equation of the form of Eq. (3.1). Note that this approximate solution is the same for the projection operator and Redfield approaches. In the absence of an external field, or one constant in time, the evolution equation has a convolution structure whose solution is the same as obtained by the noninteracting blip approximation. ${ }^{8}$ For suitable spectral densities, and not too low a temperature, time-local equations result, and the evolution is characterized by a rate constant. The presence of a time-dependent external field precludes a rate process, but an average over the period of the external field should characterize the relaxation. In principle, however, it should be possible to detect a nonexponential decay in response to the oscillatory external field. In any case, once the external field's frequency becomes large relative to the relaxation time of the rate kernel [cf. Eq. (3.9)], the time variation of $\Gamma(t)$ becomes negligible and a rate process will be obtained. For sufficiently high-frequency fields, where $4 b / \hbar \Omega$ is small, the corresponding averaged rate becomes independent of the external field, as one might anticipate. Thus, scanning $\Omega$ from low to high frequency could also provide a method of controlling the tunneling rate.

The comparison of the temperature dependencies of the averaged rate, for a time-dependent external field, and the rate for a constant external field, relative to the fieldindependent value, shows that the tunnel rate can be enhanced or suppressed. The external field becomes increasingly significant as the temperature decreases because the energy parameters $\varepsilon$ and $b(t)$ are scaled relative to the temperature. The value we have chosen for $\varepsilon$, in the 1 -K range, is typical of energies found in the impurity in a metal experiment. A $4 b$ value of this order corresponds to an external field magnitude of about $1000 \mathrm{~V} / \mathrm{cm}$, if the dipole's magnitude is 1 Debye.

Finally, in this work we have tried to provide analytic expressions that illustrate the principals that can be found for the control of tunneling with external fields. This requires the use of fields of simple form, either constant or sinusoidal. At the expense of some numerical analysis, our results can also accommodate the use of fields of arbitrary shape.

\section{ACKNOWLEDGMENTS}

Support by the NATO (R.I.C. and M.M.), the Center for Fundamental Materials Research at Michigan State Univer- 
sity (R.I.C. and M.M.), and the DGICYT of Spain and the Junta de Andalucia (M.M.) is gratefully acknowledged.

\section{APPENDIX}

In this appendix we evaluate the elements of the relaxation tensor $R$. Equations (2.20b), (2.21), and (2.5)-(2.8) show that the basic quantities of interest that must be evaluated are $\Gamma_{i j k l}^{ \pm}$. They are obtained from Eqs. (2.20b) and (2.21). As $\omega_{i k}$ in our model are proportional to $\bar{V}$, these frequencies are very small and may be neglected in the expressions for $\Gamma_{i j k l}^{ \pm}$. With this approximation, we find

$$
\begin{aligned}
\Gamma_{1111}^{+} & =\Gamma_{2222}^{+}=-\Gamma_{1122}^{+}=-\Gamma_{2211}^{+} \\
& =\left\langle\Phi^{+}(t-\tau) \Phi^{+}\right\rangle \cos 2 \lambda(t, \tau), \\
\Gamma_{1212}^{+} & =\Gamma_{2121}^{+}=-\Gamma_{2112}^{+}=-\Gamma_{1221}^{+} \\
& =\left\langle\Phi^{-}(t-\tau) \Phi^{-}\right\rangle \cos 2 \lambda(t, \tau), \\
\Gamma_{1211}^{+} & =\Gamma_{2122}^{+}=-\Gamma_{2111}^{+}=-\Gamma_{1222}^{+} \\
& =\left\langle\Phi^{-}(t-\tau) \Phi^{-}\right\rangle i \sin 2 \lambda(t, \tau), \\
\Gamma_{1112}^{+} & =\Gamma_{2221}^{+}=-\Gamma_{1121}^{+}=-\Gamma_{2212}^{+} \\
& =\left\langle\Phi^{+}(t-\tau) \Phi^{+}\right\rangle i \sin 2 \lambda(t, \tau)
\end{aligned}
$$

and

$$
\begin{aligned}
\Gamma_{1111}^{-} & =\Gamma_{2222}^{-}=-\Gamma_{1122}^{-}=-\Gamma_{2211}^{-} \\
& =\left\langle\Phi^{+} \Phi^{+}(t-\tau)\right\rangle \cos 2 \lambda(t, \tau), \\
\Gamma_{1212}^{-} & =\Gamma_{2121}^{-}=-\Gamma_{2112}^{-}=-\Gamma_{1221}^{-} \\
& =\left\langle\Phi^{-} \Phi^{-}(t-\tau)\right\rangle \cos 2 \lambda(t, \tau), \\
\Gamma_{1211}^{-} & =\Gamma_{2122}^{-}=-\Gamma_{2111}^{-}=-\Gamma_{1222}^{-} \\
& =\left\langle\Phi^{+} \Phi^{+}(t-\tau)\right\rangle i \sin 2 \lambda(t, \tau), \\
\Gamma_{1112}^{-} & =\Gamma_{2221}^{-}=-\Gamma_{1121}^{-}=-\Gamma_{2212}^{-} \\
& =\left\langle\Phi^{-} \Phi^{-}(t-\tau)\right\rangle i \sin 2 \lambda(t, \tau) .
\end{aligned}
$$

In obtaining these equations we have used the results

$$
\begin{aligned}
\left\langle\Phi^{+} \Phi^{-}(t-\tau)\right\rangle & =\left\langle\Phi^{-} \Phi^{+}(t-\tau)\right\rangle=\left\langle\Phi^{+}(t-\tau) \Phi^{-}\right\rangle \\
& =\left\langle\Phi^{-}(t-\tau) \Phi^{+}\right\rangle=0 .
\end{aligned}
$$

The nonzero correlation functions are

$$
\begin{gathered}
\left\langle\Phi^{+}(t-\tau) \Phi^{+}\right\rangle=4 e^{-W(0)}[\cosh W(t-\tau)-1], \\
\left\langle\Phi^{+} \Phi^{+}(t-\tau)\right\rangle=4 e^{-W(0)}[\cosh W(\tau-t)-1], \\
\left\langle\Phi^{-}(t-\tau) \Phi^{-}\right\rangle=-4 e^{-W(0)} \sinh W(t-\tau), \\
\left\langle\Phi^{-} \Phi^{-}(t-\tau)\right\rangle=-4 e^{-W(0)} \sinh W(\tau-t),
\end{gathered}
$$

where

$$
\begin{aligned}
W(t) & =\sum_{j}\left(2 \gamma_{j}^{2} / \hbar \omega_{j}^{3}\right)\left[\operatorname{coth}\left(\beta \hbar \omega_{j} / 2\right) \cos \omega_{j} t-i \sin \omega_{j} t\right] \\
& \equiv \Psi(t)-i G(t) .
\end{aligned}
$$

Note that $W(t=0) / 2$ is the exponent in the Franck-Condon factor renormalizing the tunnel splitting [cf. Eqs. (2.7) and (2.8)]. It arises from the overlap of the medium wave functions centered around the left and right local states of the tunneling system. The quantum equilibrium time-correlation functions in Eqs. (A2) and (A3) are evaluated by expressing the time evolution of the momentum operator $p(t)$ appearing in Eq. (2.4) in terms of its initial values $q(0)$ and $p(0)$ according to the medium harmonic-oscillator dynamics of Eq. (2.4b). The resulting Gaussian average over these initial-time coordinate and momentum operators can then be obtained by standard techniques. ${ }^{33}$ It is conventional to express $W(t)$ as an integral over a spectral density $J(\omega)$ of the medium $\operatorname{modes}^{1,5,8}$ as

$$
W(t)=\int_{\omega_{\min }}^{\omega_{\max }}\left(J(\omega) / \omega^{2}\right)[\operatorname{coth}(\beta \hbar \omega / 2) \cos \omega t-i \sin \omega t] d \omega
$$

with

$$
J(\omega) \equiv \sum_{j}\left(2 \gamma_{j}^{2} / \hbar \omega_{j}\right) \delta\left(\omega-\omega_{j}\right)
$$

Note that the spectral density reflects the nature of the coupling between the system and the medium.

With these averages and Eq. (A1), the elements of the $R$ tensor can be obtained from Eq. (2.20b). They are

$$
\begin{aligned}
R_{1111}=R_{1221}= & R_{2222}=R_{2112}=-R_{1122}=-R_{2211} \\
= & -(\bar{V} / \hbar)^{2} \cos 2 \lambda(t, \tau)[\sinh W(\tau-t) \\
& +\sinh W(t-\tau)], \\
R_{1212}=R_{2121}= & -2(\bar{V} / \hbar)^{2} \cos 2 \lambda(t, \tau)[\cosh W(\tau-t) \\
& +\cosh W(t-\tau)-2]+R_{1111}, \\
R_{1211}=R_{2122}=- & (\bar{V} / \hbar)^{2} i \sin 2 \lambda(t, \tau)\{[\sinh W(t-\tau) \\
- & \sinh W(\tau-t)]-2[\cosh W(\tau-t)-1]\},
\end{aligned}
$$

$$
\begin{aligned}
R_{2111}=R_{1222}= & -(\bar{V} / \hbar)^{2} i \sin 2 \lambda(t, \tau)\{[\sinh W(t-\tau) \\
& -\sinh W(\tau-t)]+2[\cosh W(t-\tau)-1]\}
\end{aligned}
$$

$$
\begin{aligned}
R_{1112} & =R_{2221}=-R_{1121}=-R_{2212} \\
& =(\bar{V} / \hbar)^{2} i \sin 2 \lambda(t, \tau)[\sinh W(t-\tau)+\sinh W(\tau-t)]
\end{aligned}
$$

These are the explicit expressions for the five independent quantities characterizing $R$ that are used in Eq. (2.27). 
*Permanent address: Fisica Teorica, Universidad de Sevilla, Apdo, Correos 1065, Sevilla 41080 Spain.

${ }^{1} \mathrm{~J}$. Ulstrup, Charge Transfer Processes in Condensed Media (Springer, Berlin, 1979).

${ }^{2}$ R. A. Marcus and N. Sutin, Biochim. Biophys. Acta 811, 265 (1985).

${ }^{3}$ V. G. Levich, in Physical Chemistry-An Advanced Treatise, edited by H. Henderson and W. Yost (Academic, New York, 1970), Vol. 9B, p. 985.

${ }^{4}$ D. DeVault, Quantum Mechanical Tunneling in Biological Systems (Cambridge University Press, London, 1984).

${ }^{5}$ B. Fain, Theory of Rate Processes in Condensed Media (Springer, Berlin, 1980).

${ }^{6}$ A. M. Stoneham, Theory of Defects in Solids (Clarendon, Oxford, 1975).

${ }^{7}$ H. Bottker and V. V. Bryksin, Hopping Conduction in Solids (Akademic, Berlin, 1985).

${ }^{8}$ A. J. Leggett, S. Chakravarty, A. T. Dorsey, M. P. A. Fisher, A. Garg, and W. Zwerger, Rev. Mod. Phys. 59, 1 (1987).

${ }^{9}$ V. A. Benderskii, D. E. Makarov, and C. A. Wight, Adv. Chem. Phys. 88, 1 (1994).

${ }^{10}$ F. L. Carter, Molecular Electronic Devices (Dekker, New York, 1982).

${ }^{11}$ Molecular Electronic Devices II, edited by F. L. Carter (Dekker, New York, 1987).

${ }^{12}$ Molecular Electronic Devices, edited by F. L. Carter, R. E. Siatkowski, and H. Wohltjen (North-Holland, Amsterdam, 1988).

${ }^{13}$ M. Morillo and R. I. Cukier, J. Chem. Phys. 98, 4548 (1993).

${ }^{14}$ R. I. Cukier and M. Morillo, Chem. Phys. 183, 375 (1994).

${ }^{15}$ T. Dittrich, B. O. Oelschlagel, and P. Hanggi, Europhys. Lett. 22, 5 (1993).

${ }^{16}$ A. Farazdel, M. Dupuis, E. Clementi, and A. Aviram, J. Am. Chem. Soc. 112, 4206 (1990).

${ }^{17}$ F. Grossmann, T. Dittrich, P. Jung, and P. Hanggi, Phys. Rev. Lett. 67, 516 (1991).

${ }^{18}$ F. Grossmann and P. Hanggi, Europhys. Lett. 18, 571 (1992).

${ }^{19}$ F. Grossmann, T. Dittrich, P. Jung, and P. Hanggi, J. Stat. Phys. 70, 229 (1993).

${ }^{20}$ F. Grossmann, P. Jung, T. Dittrich, and P. Hanggi, Z. Phys. B 84, 315 (1991)

${ }^{21}$ Y. Dakhnovskii, Ann. Phys. (N.Y.) 229, 145 (1994).

${ }^{22}$ Y. Dakhnovskii, Phys. Rev. B 49, 4649 (1994).

${ }^{23}$ Y. Dakhnovskii, J. Chem. Phys. 100, 6492 (1994).

${ }^{24}$ Y. Dakhnovskii and R. D. Coalson, J. Chem. Phys. 103, 2908 (1995)

${ }^{25}$ Y. Dakhnovskii, D. G. Evans, H. J. Kim, and R. D. Coalson, J. Chem. Phys. 103, 5461 (1995).

${ }^{26}$ I. A. Goychuk, E. G. Petrov, and V. May, Phys. Rev. E 52, 2392 (1995).

${ }^{27}$ M. Grifoni, M. Sassetti, P. Hanggi, and U. Weiss, Phys. Rev. E 52, 3596 (1995).
${ }^{28}$ I. A. Goychuk, E. G. Petrov, and V. May, Chem. Phys. Lett. 253, 428 (1996).

${ }^{29}$ M. Grifoni, M. Sassetti, and U. Weiss, Phys. Rev. E 53, R2033 (1996).

${ }^{30}$ Molecular Electronics Biosensors and Biocomputers, edited by F. T. Hong (Plenum, New York, 1989).

${ }^{31}$ Molecular Electronics-Science and Technology, edited by A. Aviram (AIP, New York, 1992).

${ }^{32}$ Molecular Electronics, edited by G. J. Ashwell (Wiley, New York, 1992).

${ }^{33}$ A. Messiah, Quantum Mechanics (North-Holland, Amsterdam, 1966).

${ }^{34}$ R. Silbey and R. A. Harris, J. Phys. Chem. 93, 7062 (1989).

${ }^{35}$ B. Golding, N. M. Zimmerman, and S. N. Coppersmith, Phys. Rev. Lett. 68, 998 (1992).

${ }^{36}$ K. Chun and N. O. Birge, Phys. Rev. B 48, 11500 (1993).

${ }^{37}$ N. O. Birge, K. Chun, G. B. Alers, and B. Golding, Physica B 194-196, 981 (1994).

${ }^{38}$ J. Kondo, Physica B 125, 279 (1984).

${ }^{39}$ J. Kondo, Physica B 126, 377 (1987).

${ }^{40}$ K. Yamada, A. Sakurai, and S. Miyazima, Prog. Theor. Phys. 73, 1342 (1985).

${ }^{41}$ D. Richter, in Quantum Aspects of Molecular Motions in Solids, edited by A. Heidemann, A. Magerl, M. Prager, D. Richter, and T. Springer (Springer, Berlin, 1987).

${ }^{42}$ D. Steinbinder, H. Wipf, A. Magerl, D. Richter, A.-J. Dianoux, and K. Neumaier, Europhys. Lett. 6, 535 (1988).

${ }^{43}$ Y. Kagan and N. V. Prokof'ev, Zh. Eksp. Teor. Fiz. 90, 2176 (1986) [Sov. Phys JETP 63, 1276 (1986)].

${ }^{44}$ H. Grabert and U. Weiss, Phys. Rev. Lett. 54, 1605 (1985).

${ }^{45}$ H. Grabert, Phys. Rev. B 46, 12753 (1992).

${ }^{46}$ M. P. A. Fisher and A. T. Dorsey, Phys. Rev. Lett. 54, 1609 (1985).

${ }^{47}$ M. Morillo, R. I. Cukier, and M. Tij, Physica A 179, 411 (1991).

${ }^{48}$ M. Morillo and M. Tij, Physica A 179, 428 (1991).

${ }^{49}$ A. O. Caldeira and A. J. Leggett, Ann. Phys. 149, 374 (1983).

${ }^{50}$ M. Eckert and G. Zundel, J. Phys. Chem. 91, 5170 (1987).

${ }^{51}$ R. Janoschek, E. G. Weidemann, H. Pfeiffer, and G. Zundel, J. Am. Chem. Soc. 94, 2387 (1972).

${ }^{52}$ E. G. Weidemann and G. Zundel, Z. Naturforsch. 25A, 627 (1970).

${ }^{53}$ K. G. Petzinger, Phys. Rev. B 26, 6530 (1982).

${ }^{54}$ V. Romero-Rochin and I. Oppenheim, Physica A 155, 52 (1989).

${ }^{55}$ R. Zwanzig, J. Chem. Phys. 33, 1338 (1960).

${ }^{56}$ N. G. van Kampen, Stochastic Processes in Physics and Chemistry (North-Holland, Amsterdam, 1981).

${ }^{57}$ A. G. Redfield, Adv. Magn. Res. 1, 1 (1965).

${ }^{58}$ J. Kondo, Physica B 84, 40 (1976).

${ }^{59}$ S. Chakravarty and J. Rudnick, Phys. Rev. Lett. 75, 501 (1995). 